\title{
Enhanced tribological properties of lotus leaf-like surfaces fabricated by capillary force lithography
}

\author{
R. A. Singh ${ }^{1}$, E.-S. Yoon*1, H. J. Kim¹, H. Kong ${ }^{1}$, S. Park ${ }^{2}$, H. E. Jeong ${ }^{3}$ and \\ K. Y. Suh ${ }^{3}$
}

The surface topography of a real lotus leaf was successfully replicated using a capillarity directed soft lithographic technique. The replication was carried out on poly(methyl methacrylate) (PMMA) film coated on a silicon wafer. The friction property of the patterned surfaces was investigated on a microscale. Its friction coefficient was almost six times lower than that of the PMMA thin film. The reduced real area of contact projected by the patterned surface appears to be the main reason for its superior friction property.

Keywords: Biomimetic, Lithography, Polymer, Tribology

\section{Introduction}

In the last few years, the concept of 'Lotus effect' has motivated scientists worldwide to modify/fabricate surfaces for real applications. ${ }^{1-3}$ Motivated by the surface topography of lotus leaf, tribologists have modified/ fabricated surfaces by means of laser texturing, ion beam roughening and fabrication of biomimetic nanopatterns in order to enhance the tribological performance on a micro-/nanoscale. ${ }^{4-8}$ Here, the authors report the replication of a real lotus leaf surface by a simple capillarity directed soft lithographic technique and the superior tribological property shown by the surface on a microscale. To the authors' knowledge, this is the first report on creating an artificial surface using a natural template by the process of capillarity directed soft lithography and its investigation in the context of tribology.

\section{Experimental}

The replication of a lotus leaf surface was carried out on poly(methyl methacrylate) (PMMA) film coated on silicon wafer, using a simple capillarity directed soft lithography technique, which principally utilises the competition between capillary and hydrodynamic forces in the course of pattern formation. ${ }^{9}$ The patterned surface so fabricated was investigated for its microfriction property and was compared with that of the PMMA thin film and silicon wafer. It is worthwhile to note here that in micro-/nanoscale devices such as micro-/nanoelectromechanical systems (MEMS/NEMS),

${ }^{1}$ Tribology Research Center, Korea Institute of Science and Technology, Seoul 130-650, Korea

${ }^{2}$ Microsystem Research Center, Korea Institute of Science and Technology, Seoul 130-650, Korea

${ }^{3}$ School of Mechanical and Aerospace Engineering, Seoul National University, Seoul 151-742, Korea

*Corresponding author, email esyoon@kist.re.kr silicon is a traditionally used material and PMMA is a polymer often found in these devices. ${ }^{10}$ Hence, from the tribological point of view, a comparison between the performance of the patterned surface and these two materials becomes important. Friction tests were performed using glass balls of $0.5 \mathrm{~mm}$ radius (normal load $3 \mathrm{mN}$, sliding speed $1 \mathrm{~mm} \mathrm{~s}^{-1}$, scan length $3 \mathrm{~mm}$ ) in a ball on flat type microtribotester under reciprocating motion, ${ }^{11}$ in controlled conditions of temperature $\left(24 \pm 1^{\circ} \mathrm{C}\right)$ and relative humidity $(45 \pm 5 \%)$. Tests were repeated more than five times and the average values were plotted.

Figure 1 shows a schematic diagram of the fabrication procedure of the patterned surface. As with the first step, a UV cured mould, polyurethane acrylate (PUA) mould, was made using a real lotus (Nelumbo nucifera) leaf as the natural template. The PUA mould was composed of a functionalised prepolymer with acrylate groups for crosslinking, a monomeric modulator, a photoinitiator and a radiation curable releasing agent for surface activity. ${ }^{12}$ This liquid mixture was drop dispensed onto a leaf of lotus. A transparent polyethylene terephthalate (PET) film was placed on the liquid mixture, followed by UV $(\lambda=250-400 \mathrm{~nm})$ exposure for a few tens of seconds. After the UV curing, the mould was peeled off from the leaf. Next, PMMA, dissolved in toluene (15 wt- $\%$ ), was spin coated onto the cleaned silicon wafer. Finally, the PUA mould was placed on the PMMA surface under a slight pressure of $\sim 980 \mathrm{~Pa}$ for conformal contact with the polymer. For a uniform pressure distribution, a thin poly (dimethyl siloxane) (PDMS) block was placed as a buffer on the top of the PUA mould before the application of pressure. The sample was thus annealed at $130^{\circ} \mathrm{C}$, well above the glass transition temperature, for $30 \mathrm{~min}$ on a hot stage, after which the PUA mould was removed.

\section{Results and discussion}

Figure $2 a$ and $b$ show the surface of a real lotus (Nelumbo nucifera) leaf at two different magnifications 

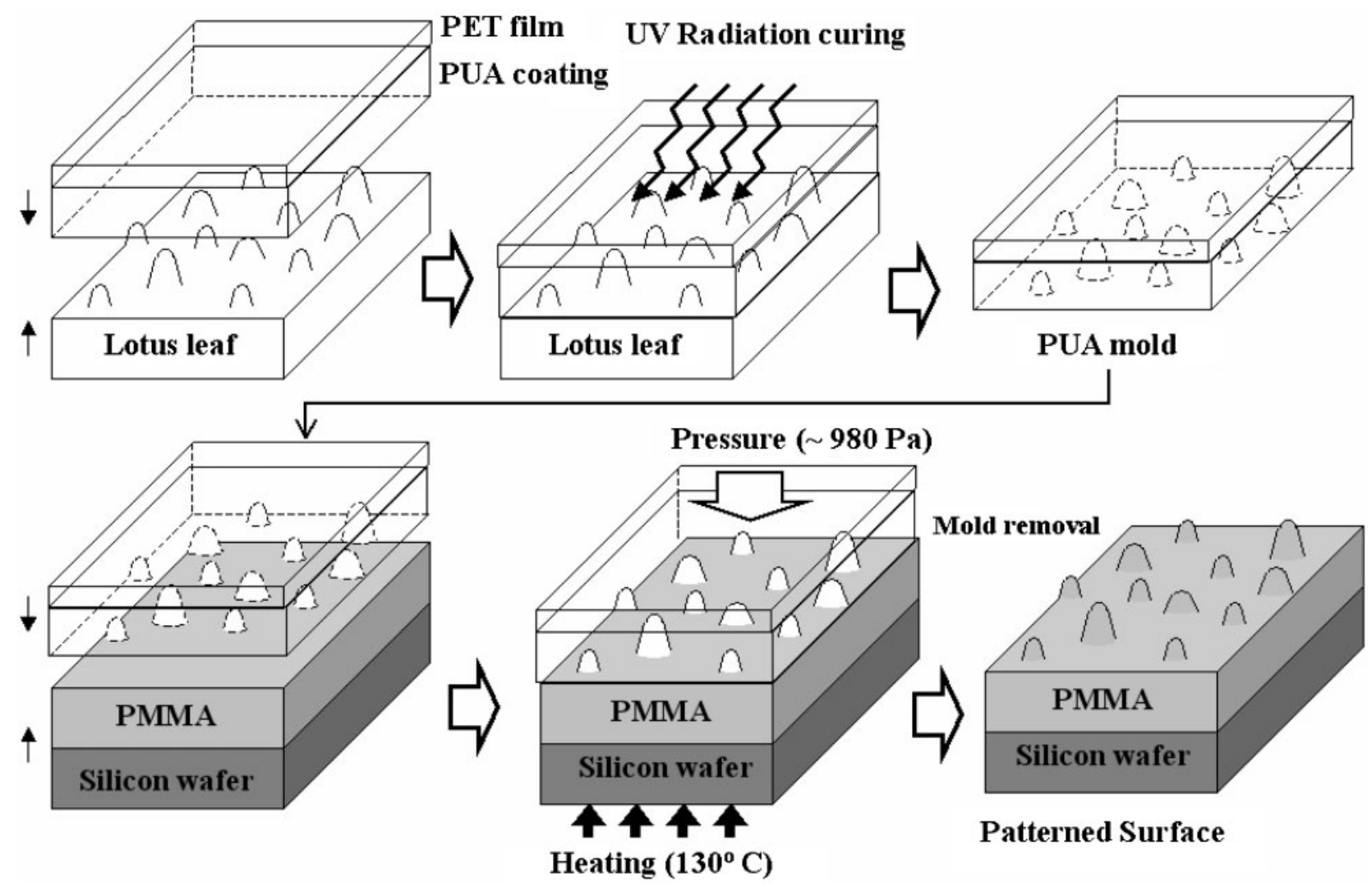

Patterned Surface

1 Schematic diagram of moulding procedure using lotus leaf as natural template and fabrication of lotus leaf-like surface using capillarity directed soft lithographic technique

while Fig. $2 c$ and $d$ show the patterned PMMA surface. It can be seen that the lotus leaf surface has been replicated successfully on a smoother scale, with the features of protuberances on the wavy surface. The PMMA thin film and the patterned surface were characterised for their static contact angle of water using the sessile drop method. It was found that the water contact angle of the PMMA thin film was $69^{\circ}$ and that of the patterned surface was $106^{\circ}$. The water contact angle of a silicon wafer is $\sim 22^{\circ} .{ }^{11}$ A real lotus

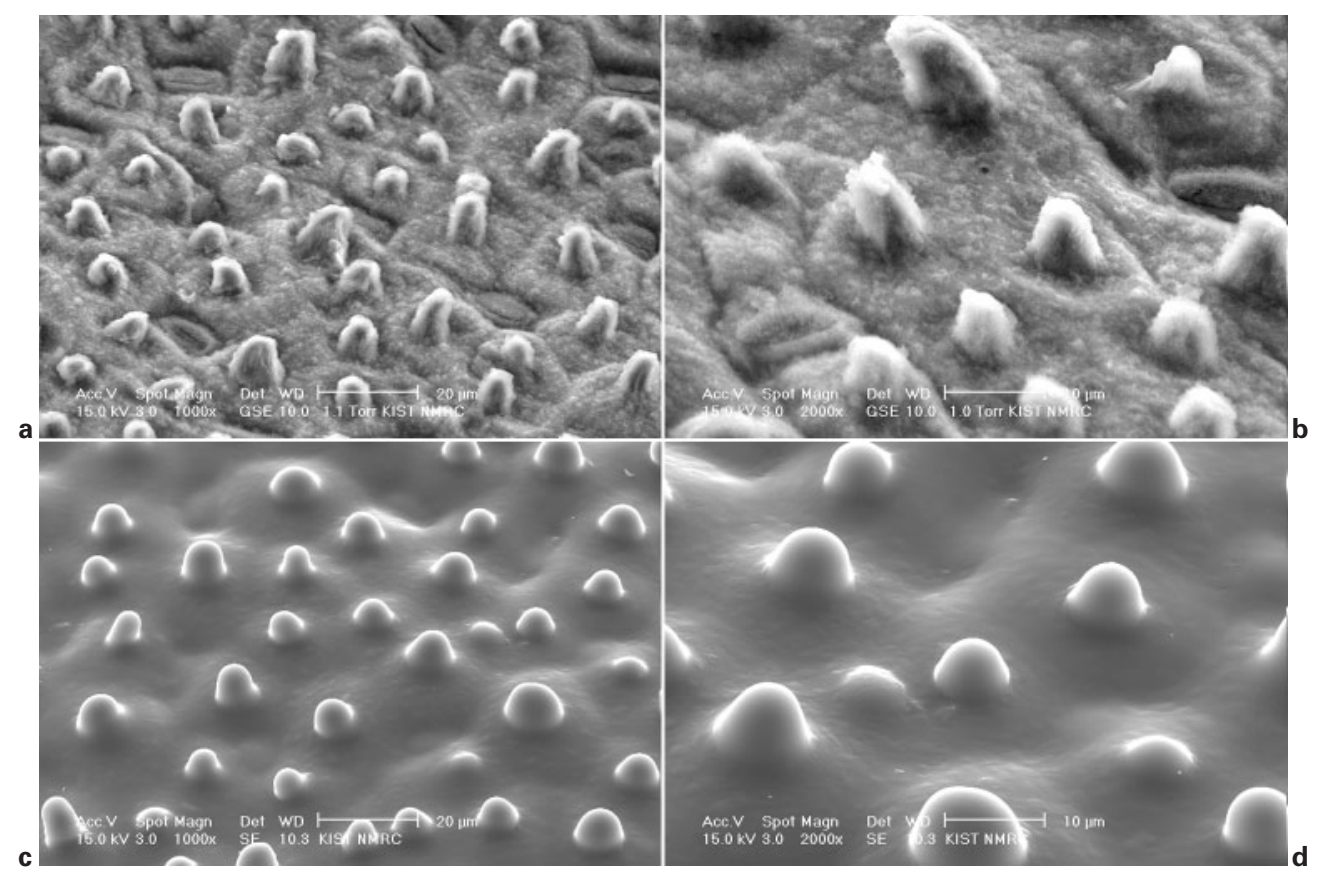

$a, c \times 1000 ; c, d \times 2000$

2 Scanning electron microscopy images of lotus (Nelumbo nucifera) leaf and patterned PMMA surface at two different magnifications 


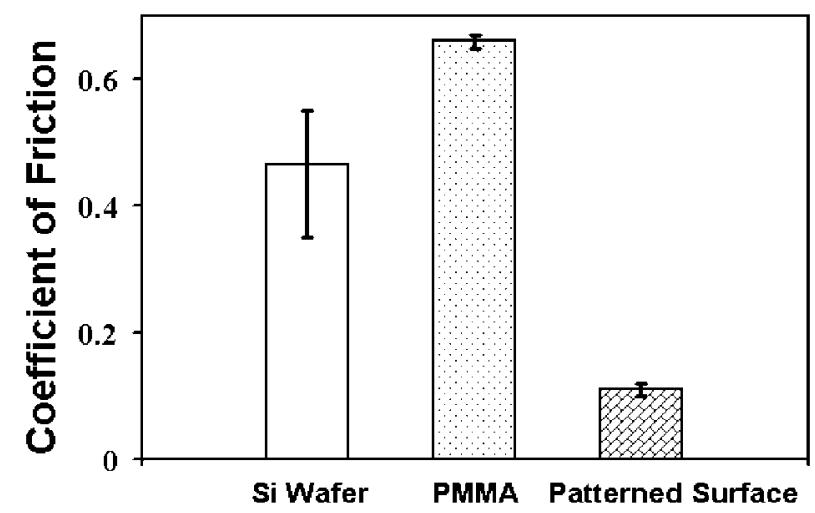

3 Coefficient of friction of test samples: patterned surface shows superior friction property when compared with PMMA thin film and silicon wafer

leaf has a water contact angle value of $\sim 162^{\circ},{ }^{13}$ which is due to the combination of the protuberances and the wax on it.

Figure 3 shows the results of the microfriction tests. The patterned surface showed a coefficient of friction, which is almost six times lower than that of the PMMA thin film and four times lower than that of the silicon wafer. According to the fundamental law of friction given by Bowden and Tabor, ${ }^{14}$ friction force is directly proportional to the real area of contact. In the present case, the patterned surface shows a lower friction value, when compared with the PMMA thin film, owing to its reduced real area of contact. This is due to the fact that the patterning of a surface causes a reduction in the real area of contact when the size of the asperities (protuberances) is considerably smaller than that of the counterface slider (glass ball in the present case). ${ }^{6,15}$ Furthermore, considering JKR theory, the contact area depends not only on the applied normal load and the radius of the ball, but also on the interfacial energy. ${ }^{16} \mathrm{It}$ is well known that the cosine of water contact angle is a measure of surface energy. ${ }^{17}$ When compared with the PMMA thin film and silicon wafer, the patterned surface has a lower surface energy, which is indicated by its higher value of water contact angle. Hence, the lower surface energy of the patterned surface further contributes towards reducing the real area of contact thereby lowering its friction value. ${ }^{11,17,18}$

Figure 4 shows the representative surfaces of silicon wafer, PMMA thin film and the patterned surface after the friction tests. The silicon wafer and PMMA thin film undergo wear whereas the patterned surface shows roughening and deformation at the tip of the protuberances. Furthermore, microscale friction tests of patterned surfaces with various geometrical parameters, fabricated using an artificial template by the same soft lithographic method (under the same experimental conditions as those in the present investigation), ${ }^{7,8}$ showed that the lowest possible value of coefficient of friction was about one-third of the PMMA thin film. ${ }^{7,8}$ This finding indicates that the surface of a lotus leaf appears to be highly optimised in terms of friction despite the fact that one might be able to create a low friction surface using artificial templates with carefully designed parameters.

On a comparative note between various surface modification/fabrication methods mentioned earlier

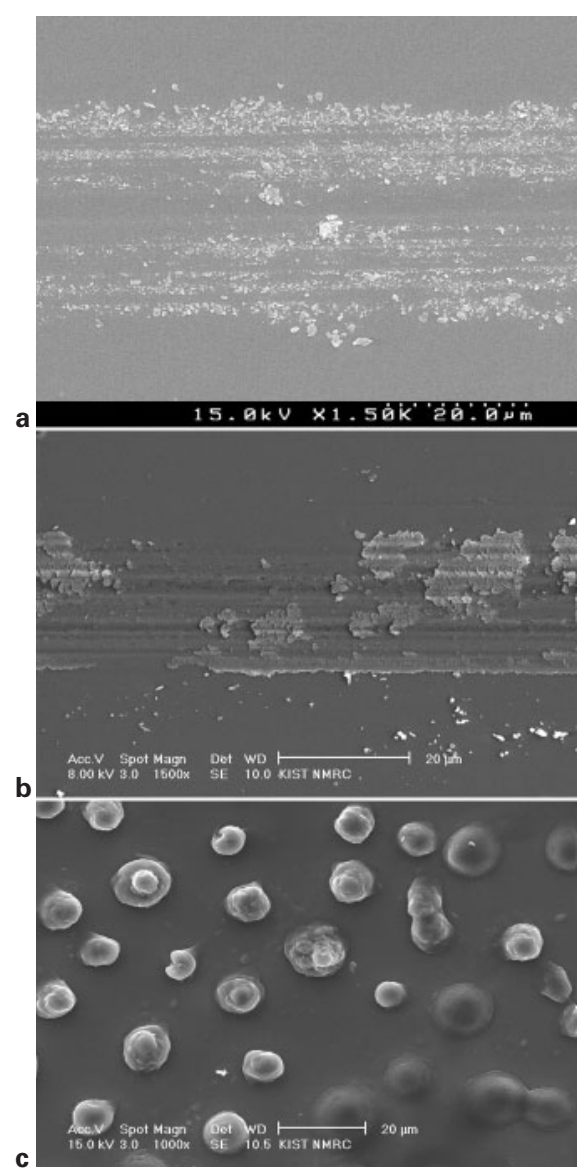

4 a worn surface of silicon wafer, $b$ wear track on PMMA thin film and $c$ roughened and deformed protuberances in case of patterned surface on sliding

such as laser texturing and ion beam roughening ${ }^{4,5}$ and the present topographical patterning, the latter method is simple, cost effective, and less time consuming. As a part of continuing research work, the authors are exploring the replication of other natural surfaces with different polymeric materials to ensure flexibility of the fabrication method, which would provide a wide range of surfaces with superior tribological properties.

\section{Conclusions}

In conclusion, a simple and cost effective method for fabricating a patterned surface has been presented by direct replication of a natural surface and its potential as a tribological candidate highlighted.

\section{Acknowledgements}

This research work was supported by a grant (05K140100930) from Center for Nanoscale Mechatronics and Manufacturing of 21st Century Frontier Research Program. This work was also supported in part by the Micro Thermal System Research Center of Seoul National University.

\section{References}

1. P. Gould: Mater. Today, Nov. 2003, 44-48.

2. H. Y. Erbil, A. L. Demirel, Y. Avci and O. Mert: Science, 2003, 299, 1377-1380.

3. M. Sun, C. Luo, L. Xu, H. Ji, Q. Ouyang, D. Yu and Y. Chen: Langmuir, 2005, 21, 8978-8981. 
4. Y. Ando and J. Ino: J. Tribol., 1997, 119, 781-787.

5. E. S. Yoon, S. H. Yang, H. Kong and K. H. Kim: Tribol. Lett., 2003, 15, 145-154

6. Z. Burton and B. Bhushan: Nano Lett., 2005, 5, 1607-1613.

7. E. S. Yoon, R. A. Singh, H. Kong, B. Kim, D. H. Kim, K. Y. Suh and H. E. Jeong: Proc. World Tribology Congress III, Washington, DC, USA, September 2005, Washington Hilton, Paper Number WTC2005-63964.

8. E. S. Yoon, R. A. Singh, H. Kong, B. Kim, D. H. Kim, K. Y. Suh and H. E. Jeong: Tribol. Lett., 2006, 21, 31-37.

9. K. Y. Suh, Y. S. Kim and H. H. Lee: Adv. Mater., 2001, 13, 1386 1389.

10. S. T. Nikhil and B. Bhushan: Nanotechnology, 2004, 15, 15611570 .
11. E. S. Yoon, R. A. Singh, H. J. Oh and H. Kong: Wear, 2005, 259, 1424-1431.

12. S. J. Choi, P. J. Yoo, S. J. Baek, T. W. Kim and H. H. Lee: J. Am. Chem. Soc., 2004, 126, 7744-7745.

13. C. Neinhuis and W. Barthlott: Ann. Bot., 1997, 79, 667-677.

14. F. P. Bowden and D. Tabor: 'Friction and lubrication'; 1956, London, Methuen.

15. M. Zou, L. Cai, H. Wang, D. Yang and T. Wyrobek: Tribol. Lett., 2005, 20, 43-52.

16. K. L. Johnson, K. Kendall and A. D. Roberts: Proc. R. Soc. Lond. A, 1971, 324A, 301-313.

17. B. D. Beake and G. J. Leggett: Langmuir, 2000, 16, 735-739.

18. R. A. Singh, E. S. Yoon, H. G. Han and H. Kong: Wear, in press (on line June 2006). 